Asian Journal of Medical Sciences 10(2): 13-18, 2019

DOI:10.19026/ajms.10.6022

ISSN:2040-8765; e-ISSN: 2040-8773

(C) 2019 Maxwell Scientific Publication Corp.

Submitted: December 13, 2018

Accepted: March 6, 2019

Published: July 25, 2019

\title{
Research Article \\ The Effects of Chronic Kidney Disease on Liver Function Tests: The Laboratory Interpretation Perspective
}

\author{
${ }^{1}$ Wilfred Kimani Gatua, ${ }^{2}$ Joseph J.N. Ngeranwa and ${ }^{3}$ Patrick Mbugua \\ ${ }^{1}$ Department of Laboratory Medicine, Kenyatta National Hospital, \\ ${ }^{2}$ Department of Biochemistry and Biotechnology, Kenyatta University, \\ ${ }^{3}$ Department of Medicine, Renal Unit, Kenyatta National Hospital, Kenya
}

\begin{abstract}
The aim of the study was to determine the levels of routinely measured liver function tests such as liver enzymes (AST, ALT, ALP, GGT \& LDH), serum proteins (albumin and total) and serum bilirubins in patients with Chronic Kidney Disease (CKD). Clinical management of CKD involves frequent laboratory monitoring of liver function tests. It is therefore important to understand the levels of these biochemistry parameters in both healthy individuals and those with CKD for easier and accurate interpretation. A cross-sectional study of two groups of study subjects including patients with CKD and control group was used. A total of 180 subjects with CKD were recruited for the study plus another 200 healthy subjects who served as the control group and were recruited from among blood donors. About $2.0 \mathrm{~mL}$ of venous blood specimen was removed from each patient and from control subjects. Levels of the enzymes Aspartate Transaminase (AST), Alkaline Phosphatase (ALP), Alanine Transaminase (ALT), Gamma Glutamyl Transferase ( $\gamma$-GT) and Lactate Dehydrogenase (LDH) were measured. Levels of serum protein and bilirubin were also determined in the two groups of study subjects. The Kolmogrov-Smirnov test was used to test the data for normality. The data obtained was parametric in nature and was tabulated as a mean and standard deviation. Parametric method of data analysis was used. Descriptive statistics used included parameters of location and parameters of dispersion. Means differences between investigated groups (control and chronic kidney disease patients) were assessed by ANOVA and post ANOVA statistical analysis. There were statistically significant differences $(p<0.05)$ in the levels of serum protein and liver enzymes between patients with CKD and control group but no significant differences in the levels of serum bilirubin $(\mathrm{p}>0.05)$. The results suggest that those patients with CKD exhibited low levels of AST and ALT while the levels of ALP, GGT, and LDH were relatively higher. The levels of serum proteins were relatively lower in patients with CKD as compared to the control group. There is, therefore the need for a keen interpretation of liver function tests in patients suffering from CKD.
\end{abstract}

Keywords: Bilirubin, chronic kidney disease, liver enzymes, liver function tests, serum proteins

\section{INTRODUCTION}

In chronic kidney disease the filtration capacity of the kidney is progressively reduced with consequences of anuria. Some of the causes of CKD include diabetes mellitus, hypertension, toxic substances and Systemic Lupus Erythematosus (SLE) (Kovesdy, 2012). Clinical chemistry parameters that are used to monitor the progress of disease include liver function tests (total protein, albumin, AST, ALT, ALP, $\gamma$-GT, LDH and bilirubin). Chronic Kidney Disease (CKD) consists of a wide spectrum of conditions associated with a progressive decline in kidney functions and abnormal Glomerular Filtration Rate (GFR) (Bargman and Skorecki, 2008). According to the recent guidelines of the National Kidney Foundation (Kidney Dialysis Outcomes Quality Initiatives), CKD is classified into 5 stages based on estimated GFR (eGFR) (National Kidney Foundation, 2002). Stage 1 refers to eGFR $\geq 90$ $\mathrm{mL} / \mathrm{min} / 1.73 \quad \mathrm{M}^{2}$ along with demonstrable kidney damage such as persistent proteinuria and haematuria. Stages 2, 3 and 4 corresponds to eGFR of $60-89$ $\mathrm{mL} / \mathrm{min} / 1.73 \mathrm{M}^{2}, 30-59 \mathrm{~mL} / \mathrm{min} / 1.73 \mathrm{M}^{2}$ and $15-29$ $\mathrm{mL} / \mathrm{min} / 1.73 \mathrm{M}^{2}$ respectively (Botev and Mallie, 2008)).

The main aim of treatment in these four stages of CKD is to slow down the progression of CKD to avoid complications such as cardiovascular disease. Chronic kidney disease stage 5 corresponds to eGFR of $<15$ $\mathrm{mL} / \mathrm{min} / 1.73 \mathrm{M}^{2}$ and is also known as End Stage Renal Disease (ESRD). In this stage, due to the accumulation of electrolytes, toxins and fluids, death can occur unless treated by Renal Replacement Therapy (RRT). Renal replacement therapy includes either dialysis or renal 
transplant (Perico et al., 2009). In patients with CKD, the most common associated chronic liver diseases are hepatitis B and C (Hrstic and Ostojic, 2011). In an Indian study with 134 patients on haemodialysis, 5.9\% turned out to be Hepatitis C Virus (HCV) positive whereas $1.4 \%$ of the patients had Hepatitis B Virus (HBV) infection. A dual infection was seen in 3.7\% of the patients. Another study showed that the prevalence of $\mathrm{HCV}$ infection among dialysis patients ranged between $0.7-18.1 \%$ in different Asian-Pacific countries, whereas the prevalence of $\mathrm{HBV}$ infection ranged between 1.3-14.6\% (Fabrizi et al., 2010). Added to these, is the burden of alcoholic liver diseases. Therefore, CKD patients often needs be regularly monitored with liver functions tests particularly serum levels of liver enzymes for their concomitant liver diseases (Perico et al., 2009).

Serum levels of enzymes such as Alanine Aminotransferase (ALT), Aspartate Aminotransferase (AST), Alkaline Phosphatase (ALP), Gamma Glutamyl Transferase (GGT) and lactic dehydrogenase LDH are commonly used to assess and monitor hepatic diseases. Previous studies have reported that the levels of serum aminotransferases commonly fall near the lower end of the range of normal values in patients with CKD (Fabrizi et al., 2001). Studies among hemodialysis patients also show a similar decrease in serum aminotransferases (Sette and Lopes, 2015). Thus, recognition of liver damage in CKD patients poses a challenge to laboratory medicine specialists and is hampered by the reduction in aminotransferases leading to underestimation of the disease load (Sette and Almeida Lopes, 2014). In this regard, it has been suggested that serum aminotransferases cut-off values be modified for screening for viral hepatitis in patients undergoing peritoneal dialysis.

On the other hand, plasma ALP levels can originate from liver, bone, intestine and placenta. In general, the isoenzymes from liver and bone contribute to the majority of the circulating enzyme levels. Therefore, in a patient with a liver disease, serum ALP level is an important marker for screening and monitoring. However, in a CKD patient, renal osteodystrophy could result in a significant increase in the bone isoenzymes of ALP contributing to high serum ALP level (Kovesdy et al., 2010).

Elevated GGT activity is a well-known biological marker of excessive alcohol consumption or liver injury including fatty degeneration. The enzyme has also been demonstrated to predict the development of Cardiovascular (CV) risk factors such as hypertension (Kawamoto et al., 2008) and diabetes mellitus (Chen et al., 2010). These findings suggest the possible relevance of elevated serum GGT activity in the development of Chronic Kidney Disease (CKD) because these $\mathrm{CV}$ risk factors are also known to contribute to the development of CKD. Lactate
Dehydrogenase (LDH) is usually elevated in conditions related to cardiovascular disorders and in tissue hypo perfusion as well as in dehydration.

The objective of this study was to investigate and compare the levels of serum proteins (albumin and total protein), liver enzymes (AST, ALT, ALP, GGT and LDH) as well as serum bilirubin (Total and direct bilirubin) between patients with chronic kidney disease and control group. This will go a long way in determining critical values when interpreting liver function tests in patients with chronic kidney disease in order to avoid unnecessary misinterpretation of results.

\section{MATERIALS AND METHODS}

The study was a cross-sectional, prospective study involving patients attending Renal Clinic and those admitted in Kenyatta National Hospital (KNH) with a diagnosis of Chronic Kidney Disease (CKD). Control group subjects were recruited among the blood donors at the KNH Blood Transfusion Unit (BTU). Kenyatta National Hospital is a national referral hospital with an in-patient bed capacity of about 2,000. The study was composed of two groups of study subjects; control group and patients diagnosed with chronic kidney disease. The sample size was calculated using the formula given by Lwanga et al. (1986). The study involved a total of 180 subjects with diagnosis of CKD and 200 individuals as control group.

The study consent was sort from the Kenyatta National Hospital-University of Nairobi Ethical and Research Committee (KNH/UON-ERC). Any recruited healthy individual who was found to have pathological disorders in the course of the study were advised to seek medical attention. The clinician would explain the medical disorder thereof and fill a referral and refer for further review, investigations and management. A clinician assessed and examined the subjects to determine the inclusion and exclusion criteria where medical history and clinical examination was required. The subjects were given information pertaining to ethical issues of the study in a language they could understand. They were requested to consent to the study by signing consent form. For very sick subjects, a relative was informed of the benefits of the study and requested to sign on behalf of the patient. All the individuals were asked to participate in the study on a voluntary basis after a brief explanation of the aims and purpose of the study. Those who accepted to participate were subjected to an interview using a questionnaire. Subjects above the age of 18 years who were not obese, hypertensive, pregnant, or under any medication and not taking any oral contraceptives, non-alcohol and tobacco users were recruited as control group. They were also excluded from the study if they tested positive for HIV, Hepatitis B, Hepatitis C and syphilis. Patients with a diagnosis of chronic kidney disease and 
Asian J. Med. Sci., 10(2): 13-18, 2019

Table 1: Demographic characterization of the study population

\begin{tabular}{lllll}
\hline Diagnosis & Sex & $\mathrm{N}$ & $\begin{array}{l}\text { Percent of total } \\
\text { population }\end{array}$ & $\begin{array}{l}\text { Age (years) Mean } \pm \text { S.D. } \\
\text { p }=0.463\end{array}$ \\
\hline Chronic kidney disease & Male & $108(60 \%)$ & $51 \%$ & $53 \pm 15.6$ \\
& Female & $72(40 \%)$ & & $52 \pm 12.6$ \\
Control subjects & Male & $101(50.5 \%)$ & & \\
& Female & $99(49.5 \%)$ & & \\
\hline
\end{tabular}

S.D.: Standard deviation

Table 2: Levels of liver and pancreas biomarkers in various groups of study subjects

\begin{tabular}{lll}
\hline Parameter/study subjects & Control N $=200$ & Chronic kidney disease N $=180$ \\
\hline & Mean $($ S.D. & Mean $($ S.D. \\
Albumin $(\mathrm{g} / \mathrm{L})$ & $41 \pm 5.6$ & $29 \pm 6.6^{\mathrm{a}}$ \\
Total protein $(\mathrm{g} / \mathrm{L})$ & $72 \pm 5.6$ & $60 \pm 10.4^{\mathrm{a}}$ \\
Aspartate transaminase $(\mathrm{U} / \mathrm{L})$ & $20.6 \pm 7.1$ & $17.5 \pm 6.7^{\mathrm{a}}$ \\
Alanine transaminase $(\mathrm{U} / \mathrm{L})$ & $20.6 \pm 7.1$ & $18.8 \pm 8.6^{\mathrm{a}}$ \\
$\gamma$-Glutamyl transferase $(\mathrm{U} / \mathrm{L})$ & $30.7 \pm 11.4$ & $63.3 \pm 47.9^{\mathrm{a}}$ \\
Alkaline phosphatase $(\mathrm{U} / \mathrm{L})$ & $64.4 \pm 12.4$ & $192.3 \pm 91.4^{\mathrm{a}}$ \\
Direct bilirubin $(\mu \mathrm{mol} / \mathrm{L})$ & $7.4 \pm 2.2$ & $7.1 \pm 2.7$ \\
Total bilirubin $(\mu \mathrm{mol} / \mathrm{L})$ & $11 \pm 3.4$ & $10 \pm 3.4$ \\
Lactate dehydrogenase $(\mathrm{U} / \mathrm{L})$ & $128 \pm 37$ & $226 \pm 115^{\mathrm{a}}$ \\
\hline S.
\end{tabular}

S.D.: Standard deviation; Results are expressed as mean \pm S.D.; Values with superscript a are comparisons between control group and CKD individuals $(\mathrm{p}<0.001)$

were above 18 years were recruited for the study. Eligible subjects were then requested to sign a consent form. They were required to fill a self-administered questionnaire on social and demographic data.

After application of the mentioned criteria, 180 study subjects were selected to participate in the present study. They were group as follows:

- Chronic kidney disease patients consisting of 180 subjects (108 males and 72 females) admitted in the Medical Wards or attending Renal clinic and those on dialysis at the Kenyatta National Hospital; age range was $30-65$ years, with $53 \pm 15.6$ years as mean \pm S.D.

- Control group consisting of 200 (101 males and 99 females) voluntary blood donors at Kenyatta National Hospital with a mean age of $52 \pm 12.6$ years. About $2.0 \mathrm{~mL}$ of venous blood specimen was collected from each study subject. In the laboratory, automated testing using Biolis 50i Clinical Chemistry Analyzer was done. The principle utilized in the analysis was spectrophotometric.

\section{RESULTS}

The total number of patients used in the study who had chronic kidney disease was 180 and another 200 subjects as control group. The mean age of patients with CKD was $53 \pm 15.6$ years while the mean age for the control group was $52 \pm 12.6$ years and there was no significant difference in the age distribution between the patients and control group $(p=0.463)$ as indicated in Table 1.

Levels of liver biomarkers in various groups of study subjects: Table 2 show the measured levels of albumin, total protein, AST, ALT, GGT, ALP, direct bilirubin, total bilirubin and $\mathrm{LDH}$ in the control and
CKD individuals. The levels of albumin and total protein were significantly lower in CKD than in control group $(p<0.05)$. The levels for AST and ALT were significantly lower in patients with CKD while levels of GGT, LDH and ALP were significantly higher in CKD patients than in the control $(\mathrm{p}<0.05)$. There were no significant differences in the levels of direct bilirubin and total bilirubin between control individuals and patients with CKD $(p>0.05)$.

\section{DISCUSSION}

In the current study, serum protein levels (total protein and albumin) were significantly reduced in patients with CKD. Alpha and beta globulins as well as plasma albumin are some of the proteins manufactured by the liver while gamma globulins which are used to make antibodies are manufactured by the immune system. One of the major functions of the kidney in the body is to preserve proteins. A dysfunctional kidney loses the ability to preserve proteins and a lot of it is subsequently lost through the urine with resultant reduction in the levels of protein in plasma in patients with CKD. Oedema is a common finding in patients with CKD due to the fact that in addition to fluid overload, there is also reduced oncotic pressure caused by low levels of albumin in blood. Eustace et al. (2004) showed that patients with chronic kidney disease had low plasma albumin levels. This reduced reduction in plasma protein causes water to remain in the tissues instead of moving to the vascular system, thereby causing oedema associated with kidney failure.

Most liver enzymes are found within the cytoplasm of cells at much higher concentrations than in plasma. This is due to the fact that these intra-cellular enzymes are required to catalyze metabolic reactions that are required for physiological functions. Excessive amounts of enzymes may escape into the extra-cellular space as a result of increased cell proliferation as may occur 
when there is cancer, or during cell damage due to disease. Normal plasma levels reflect the balance between the synthesis and release of enzymes during ordinary cell turn-over and their clearance from the circulation. Increased production of cells, increase in the rate of cell turn-over and cell damage usually results in raised plasma levels of enzymes. These enzymes are used as biomarkers to detect and localize increased cell damage or proliferation. Plasma enzyme levels depend on: rate of release from the cells, in turn dependent on the rate of cell damage; or the degree of proliferation or induction of enzyme synthesis.

In the current study, patients with CKD had relatively low levels of plasma aminotransferases (AST and ALT) as compared to control group. According to a study done by Sette and Lopes (2015) and Ray et al. (2015), the AST and ALT serum levels of patients with chronic kidney disease were inversely proportion to the progress of the disease (the lower the serum levels of enzymes, the severe the disease). Sette and Lopes (2015) also conducted a study which demonstrated that these enzymes were negatively correlated with creatinine levels and directly correlated with glomerular filtration rate. Some hypotheses have been given to explain the reduction of serum aminotransferases levels among patients with CKD undergoing haemodialysis. According to Lopes et al. (2009); inadequate levels of pyridoxine and blood hemodilution as well as high levels of homocystein have been given to explain the reasons for the low levels. Another study by Ono et al. (1995) on patients with CKD and who were undergoing haemodialysis had low levels of pyridoxine which could also explain the reason for low AST and ALT levels.

In the current study, there were high levels of the enzymes GGT in CKD patients as compared to control group. According to a study done by Cain et al. (2012), it was demonstrated that levels of GGT are usually elevated in CKD patients. A study by Ryu et al. (2007) indicated that serum GGT could be a biomarker for likely development of CKD. The enzyme GGT has been known to be a marker of oxidative stress. Patients with diabetes mellitus and hypertension, which are risk factors for chronic kidney disease, are known to have cellular oxidative stress. However, it is hard to draw conclusions whether serum GGT can be directly associated with CKD due to the fact that there are few studies conducted on GGT serum levels in patients with CKD undergoing haemodialysis.

Also in the current study, there were high levels of the enzymes ALP in the CKD patients as compared to control group. This is in line with a study conducted by Taliercio et al. (2013) which demonstrated higher serum ALP levels in patients with CKD stage 3 and 4. Other studies have confirmed the same findings (Regidor et al., 2008; Blayney et al., 2008; Shantouf et al., 2009). Another study by Beddhu et al. (2009) demonstrated high levels of ALP in patients with CKD which concur very well with the current study. Patients with CKD have higher ALP due to disturbances in the bone mineral disease which may contribute to the higher cardiovascular burden seen in this population. These findings, along with previous studies suggest that clinicians may use ALP as a risk assessment tool to identify patients with higher risk for ESRD progression.

The levels of LDH were higher in patients with CKD as compared to control group. In 1991, Kang et al. studied the changes of LDH activity in renal disease and reported that LDH has a positive correlation with blood urea nitrogen but no correlation with serum creatinine. This phenomenon is thought to be due to ultrafiltration-induced hemoconcentration, enzyme release from the formed elements within the extracorporeal circuit and complement-mediated leucocyte activation and pulmonary leucostasis. This is also supported by a study done by MohammadiKarakani et al. (2007) who studied the urine LDH levels and its relation to renal injury in diabetic patients. They found that the level of LDH excretion is high in diabetic patients compared with control group. Therefore, they concluded that LDH excretion has diagnostic validity in detecting renal damage in diabetic patients with a sensitivity of $62.5 \%$ and a specificity of $58.3 \%$. This phenomenon can be explained by the fact that patients with CKD had persistent high levels of waste products of metabolism such as urea, creatinine and uric acid. These nitrogenous wastes are toxic to the tissues which consequently cause increased cell damage that leads to cell necrosis and break down of cell membrane. Damage to the cell membrane facilitates the leakage of intra-cellular enzymes into the blood stream with subsequent elevation of plasma enzymes.

The current study did not demonstrate any significant differences in the levels of both total and direct bilirubin in the study subjects. Raised levels of bilirubin (jaundice) can only be caused by; pre-hepatic, hepatic and post-hepatic. Pre-hepatic jaundice is caused by excessive destruction of red blood cells as in cases of hemolysis. As a result, the rate of bilirubin formation is higher than the rate of conjugation and subsequent elimination from the body. Hepatic jaundice is caused by damaged liver cells as in cases of viral or induced drug hepatitis where the liver cells are unable to cope with bilirubin metabolism as required. On the other hand, post-hepatic jaundice is obstructive jaundice where the smooth flow of bilirubin after formation is interfered with. This is usually due to bile ducts obstruction either by gall stones or tumors. In CKD patients, none of the above pathological conditions were present and the reason why bilirubin levels remain within the reference values.

\section{CONCLUSION}

Careful interpretation of routine liver function tests (total protein, albumin, AST, ALT, ALP, GGT, LDH, 
total bilirubin and direct bilirubin) should be considered due to pathophysiological changes caused by chronic kidney disease. Serum proteins (albumin and total protein) are reduced while liver enzymes (ALP, GGT and $\mathrm{LDH}$ ) are elevated in patients with CKD. Aminotransminases (AST and ALT) are reduced in patients with CKD while the levels of bilirubin remain unaltered.

\section{ACKNOWLEDGMENT}

We acknowledge all the study subjects who accepted to participate in the study. We also acknowledge Kenyatta National Hospital management for allowing us to conduct the study. We appreciate the staff of CCU, Renal Unit and Medical Wards for their great work on screening of participants and collection of blood specimens for analysis. We highly appreciate good support given by Medtec LTD in facilitating the supply of reagents used in the study.

\section{REFERENCES}

Bargman, J.M. and K. Skorecki, 2008. Chronic kidney disease. In: Fauci, A.S., E. Braunwald, D.L. Kasper and S.L. Hauser (Eds.), Harrison's Principles of Internal Medicine. Mc Graw Hill, pp: 1761-1771.

Beddhu, S., X. Ma, B. Baird, A.K. Cheung and T. Greene, 2009. Serum alkaline phosphatase and mortality in African Americans with chronic kidney disease. Clin. J. Am. Soc. Nephrol., 4(11): 1805-1810.

Blayney, M.J., R.L. Pisoni, J.L. Bragg-Gresham, J. Bommer, L. Piera et al., 2008. High alkaline phosphatase levels in hemodialysis patients are associated with higher risk of hospitalization and death. Kidney Int., 74(5): 655-663.

Botev, R. and J.P. Mallie, 2008. Reporting the eGFR and its implication for CKD diagnosis. Clin. J. Am. Soc. Nephrol., 3(6): 1606-1607.

Cain, L.R., A.M. Ducatman and A. Shankar, 2012. The relationship between gamma-glutamyl transferase levels and chronic kidney disease among Appalachian adults. W. V. Med. J., 108(1): 8-13.

Chen, T., Y. Ren, Y. Liu, Y. Long, X. Zhang, H. Yu et al., 2010. Serum gamma-glutamyl transferase, ferritin and the risk of type 2 diabetes in women from a Chinese minority. Diabetes Res. Clin. Pract., 90(3): 352-357.

Eustace, J.A., B. Astor, P.M. Muntner, T.A. Ikizler and J. Coresh, 2004. Prevalence of acidosis and inflammation and their association with low serum albumin in chronic kidney disease. Kidney Int., 65(3): 1031-1040.

Fabrizi, F., P. Martin and P. Messa, 2010. Hepatitis B and hepatitis $\mathrm{C}$ virus and chronic kidney disease. Acta Gastroenterol. Belg., 73(4): 465-471.
Fabrizi, F., P., Martin, G. Lunghi and C. Ponticelli, 2001. Nosocomial transmission of hepatitis $C$ virus infection in hemodialysis patients: Clinical perspectives. Int. J. Artif. Organs, 23(12): 805-816.

Hrstic, I. and R. Ostojic, 2011. Chronic liver diseases in patients with chronic kidney disease. Acta Med. Croatica, 65(4): 349-353.

Kawamoto, R., K. Kohara, Y. Tabara, T. Kusunoki, N. Otsuka and T. Miki, 2008. Association between serum gamma-glutamyl transferase level and prehypertension among community-dwelling men. Tohoku J. Exp. Med., 216(3): 213-221.

Kovesdy, C.P., 2012. Metabolic acidosis and kidney disease: Does bicarbonate therapy slow the progression of CKD? Nephrol. Dial. Transplant, 27(8): 3056-3062.

Kovesdy, C.P., V. Ureche, J.L. Lu and K. KalantarZadeh, 2010. Outcome predictability of serum alkaline phosphatase in men with pre-dialysis CKD. Nephrol. Dial. Transplant., 25(9): 3003-3011.

Lopes, E.P., L.H. Sette, J.B. Sette, C.F. Luna, A.M. Andrade, M. Moraes et al., 2009. Serum alanine aminotransferase levels, hematocrit rate and body weight correlations before and after hemodialysis session. Clinics, 64(10): 941-945.

Lwanga, S.K., S. Lemeshow, W. David, Jr. Hosmer and K. Jannelle, 1986. Adequacy of sample size in healthy studies in epidemiological surveys. Bull. World Health Ooganiz., 15: 232-252.

Mohammadi-Karakani, A., S. Asgharzadeh-Haghighi, M. Ghazi-Khansari and R. Hosseini, 2007. Determination of urinary enzymes as a marker of early renal damage in diabetic patients. J. Clin. Lab. Anal., 21(06): 413-417.

National Kidney Foundation, 2002. K/DOQI clinical practice guidelines for chronic kidney disease: Evaluation, classification, and stratification. Am. J. Kid. Dis., 39: S1-266.

Ono, K., T. Ono and T. Matsumata, 1995. The pathogenesis of decreased aspartate aminotransferase and alanine aminotransferase activity in the plasma of hemodialysis patients: The role of vitamin $\mathrm{B}_{6}$ deficiency. Clin. Nephrol., 43(6): 405-408.

Perico, N., D. Cattaneo, B. Bikbov and G. Remuzzi, 2009. Hepatitis $\mathrm{C}$ infection and chronic renal diseases. Clin. J. Am. Soc. Nephrol., 4(1): 207-220.

Ray, L., S.K. Nanda, A. Chatterjee, R. Sarangi and S. Ganguly, 2015. A comparative study of serum aminotransferases in chronic kidney disease with and without end-stage renal disease: Need for new reference ranges. Int. J. Appl. Basic Med. Res., 5(1): 31-35.

Regidor, D.L., C.P. Kovesdy and R. Mehrotra, 2008. High alkaline phosphatase predicts mortality among maintenance haemodialysis patients. Clin. J. Am. Soc. Nephrol., 19(11): 2193-2203. 
Ryu, S., Y. Chang, D.I. Kim, W.S. Kim and B.S. Suh, 2007. Gamma-Glutamyltransferase as a predictor of chronic kidney disease in nonhypertensive and nondiabetic Korean men. Clin. Chem., 53(1): 7177.

Sette, L.H. and E.P. Almeida Lopes, 2014. Liver enzymes serum levels in patients with chronic kidney disease on hemodialysis: A comprehensive review. Clinics, 69(4): 271-278.

Sette, L.H. and E.P. Lopes, 2015. The reduction of serum aminotransferase levels is proportional to the decline of the glomerular filtration rate in patients with chronic kidney disease. Clinics, 70(5): 346-349.
Shantouf, R., C.P. Kovesdy, Y. Kim, N. Ahmadi, A. Luna et al., 2009. Association of serum alkaline phosphatase with coronary artery calcification in maintenance hemodialysis patients. Clin. J. Am. Soc. Nephrol., 4(6): 1106-1114.

Taliercio, J.J., J.D. Schold, J.F. Simon, S. Arrigain, A. Tang et al., 2013. Prognostic importance of serum alkaline phosphatase in CKD stages 3-4 in a clinical population. Am. J. Kidney Dis., 62(4): 703-710. 